\title{
Physical exercise improves cardiac autonomic modulation in hypertensive patients independently of angiotensin-converting enzyme inhibitor treatment
}

\author{
Izabela C Cozza ${ }^{1}$, Thaisa HR Di Sacco ${ }^{1}$, José H Mazon ${ }^{1}$, Maria Cristina O Salgado ${ }^{2}$, Sabrina GV Dutra ${ }^{1}$, \\ Evandro J Cesarino ${ }^{1}$ and Hugo CD Souza ${ }^{1}$
}

We investigated the influence of angiotensin-converting enzyme inhibitor (ACEi) treatment and physical exercise on arterial pressure (AP) and heart rate variability (HRV) in volunteer patients with hypertension. A total of 54 sedentary volunteers were divided into three groups: normotensive (NT Group), hypertensive (HT Group) and HT volunteers treated with ACEi (ACEi Group). All volunteers underwent an aerobic physical-training protocol for 15 weeks. HRV was investigated using a spectral analysis of a time series of R-R interval (RRi) that was obtained in a supine position and during a tilt test. Physical training promoted a significant reduction in the mean arterial pressure of the HT group $(113 \pm 3$ vs. $106 \pm 1 \mathrm{~mm} \mathrm{Hg})$ and the ACEi group (104 \pm 2 vs. $98 \pm 2 \mathrm{~mm} \mathrm{Hg}$ ). Spectral analysis of RRi in the supine position before physical training demonstrated that the NT and ACEi groups had similar values at low frequency (LF; $0.04-0.15 \mathrm{~Hz}$ ) and high frequency (HF; $0.15-0.5 \mathrm{~Hz}$ ) oscillations. The HT group had an increase in LF oscillations in absolute and normalized units and a decrease in HF oscillations in normalized units compared with the other groups. The HT group had the lowest responses to the tilt test during LF oscillations in normalized units. Physical training improved the autonomic modulation of the heart rate in the supine position only in the HT group. Physical training promoted a similar increase in autonomic modulation responses in the tilt test in all groups. Our findings show that aerobic physical training improves cardiac autonomic modulation in HT volunteers independently of ACEi treatment. Hypertension Research (2012) 35, 82-87; doi:10.1038/hr.2011.162; published online 29 September 2011

Keywords: aerobic physical training; angiotensin; heart rate variability

\section{INTRODUCTION}

Hypertension is highly prevalent in the adult population and represents one of the leading causes of morbidity and mortality worldwide. $^{1,2}$ This condition is directly related to the development of cardiovascular diseases, encephalic vascular accident and renal insufficiency. ${ }^{3}$ The risk factors for hypertension are associated with many other factors, including lifestyle. ${ }^{4}$ The excessive ingestion of salt and alcohol, obesity, smoking and sedentariness represent the main behavioral factors that contribute to the occurrence and development of hypertension. ${ }^{5}$

The treatment of hypertension consists mainly of a pharmacological approach, and the angiotensin-converting enzyme inhibitors (ACEis) are an important class of medications. ${ }^{6}$ ACEis reduce arterial pressure (AP) by decreasing the peripheral vascular resistance, and they reduce cardiac hypertrophy and the proliferation of vascular smooth muscle cells. ${ }^{7-9}$ Additionally, ACEis have an important role in cardiac autonomic control. Some studies have shown that ACEi treatment of hypertension in experimental animals and humans improves baroreflex sensitivity and cardiac autonomic modulation. ${ }^{10-12}$ However, these results are controversial.

Regular physical exercise has been also used for the treatment of hypertension as a co-adjuvant to pharmacological treatment and as a first intervention before prescribing of medication. ${ }^{13}$ Regular physical exercise reduces $\mathrm{AP},{ }^{14,15}$ adjusts body composition, ${ }^{16,17}$ increases aerobic capacity, ${ }^{15,16}$ controls serum fat levels ${ }^{16}$ and improves cardiac autonomic control. ${ }^{18}$

The benefits of aerobic physical training on cardiac autonomic control have been well documented in experimental and clinical studies. ${ }^{19-21}$ Regular physical exercise promotes a tonic decrease in sympathetic autonomic control and an increase in vagal control. ${ }^{22,23}$ However, the effects of physical exercise and ACEis, alone or in combination, on heart-rate variability and the capacity for autonomic balance rearrangement (for example, during the tilt test) have not been investigated in hypertensive (HT) patients. We hypothesized that the association of hypertension treatment with ACEis and physical training provides greater effects on cardiac autonomic

${ }^{1}$ Exercise Physiology Laboratory, Department of Biomechanics, Medicine and Rehabilitation, School of Medicine, University of São Paulo, Ribeirão Preto, Brazil and ${ }^{2}$ Department of Pharmacology, School of Medicine, University of São Paulo, Ribeirão Preto, Brazil

Correspondence: Dr HCD Souza, Department of Biomechanics, Medicine and Rehabilitation, School of Medicine, University of São Paulo, Ribeirão Preto, São Paulo 14049-900, Brazil.

E-mail: hugocds@fmrp.usp.br

Received 18 October 2010; revised 5 June 2011; accepted 23 June 2011; published online 29 September 2011 
modulation and enhances the reduction of arterial pressure compared with either treatment in isolation. Therefore, this study investigated the influence of physical aerobic training on arterial pressure and cardiac autonomic modulation in HT volunteers and the association between physical training and pharmacological treatment with an ACEi.

\section{METHODS}

\section{Study sample}

In all 54 non-smoking sedentary volunteers of both genders with a mean age of $44 \pm 2$ years who presented no muscular-skeletal alterations that would impede regular physical exercise were studied. The volunteers were divided into three groups: normotensive volunteers with a mean arterial pressure (MAP) of $94 \pm 3 \mathrm{~mm} \mathrm{Hg}$ (NT group; 9 women and $10 \mathrm{men}$ ), untreated volunteers with mild essential hypertension ( $\mathrm{MAP}=113 \pm 3 \mathrm{~mm} \mathrm{Hg}$ ) (HT Group; 7 women and $10 \mathrm{men}$ ), and volunteers with mild essential hypertension (MAP $=104 \pm 2 \mathrm{~mm} \mathrm{Hg}$ ) who were treated with an ACEi (Enalapril; 10-20 mg per day) for 10-15 weeks (ACEi group; 9 women and 9 men). The subjects in this latter group exhibited blood pressure levels that were similar to the HT group before treatment with Enalapril ( $\mathrm{MAP}=115 \pm 4 \mathrm{~mm} \mathrm{Hg}$ ). All subjects were informed of the study, and they read and freely signed an informed consent form. The local research ethics committee approved this study.

\section{Training protocol}

All of the volunteers submitted to an aerobic physical training protocol that consisted of a 45-min treadmill exercise three times a week for 15 weeks with a heart rate intensity of $\sim 70-80 \%$ of heart rate reserve. Aerobic capacity was re-assessed at the end of the 15-week training period.

\section{Laboratory tests and anthropometric measurements}

Serum levels of glycemia, triglycerides, total cholesterol and cholesterol fractions were measured before and after the 15 -week physical training. The volunteers were instructed to fast for $12 \mathrm{~h}$, avoid alcoholic beverage for $48 \mathrm{~h}$ before the laboratory tests, engage in no physical activity and maintain their habitual diet. The left ventricular mass index (LVM index) was calculated from the relationship between the left ventricular mass $(\mathrm{g})$ from the echocardiogram and body surface $\left(\mathrm{m}^{2}\right)$, which was calculated using the following relationship: (weight $(\mathrm{kg})+$ height $(\mathrm{cm})-60) / 100$. Body weight was measured to an accuracy of $0.1 \mathrm{~kg}$, and the body mass index was computed as body weight $/$ height $^{2}$. Tetrapolar body electrical bio-impedance determined the percent fat mass.

\section{Analysis of heart rate variability (spectral analysis)}

The volunteers were instructed to avoid alcohol and caffeine, practice no physical activity and maintain their habitual diet for $48 \mathrm{~h}$ before laboratory exams. The spectral analysis of heart rate variability (HRV) was recorded between 0900 and 1000 hours according to the following protocol: after remaining in a supine rest position on inclinable bed for $20 \mathrm{~min}$, the volunteers were passively placed in an inclined position $\left(75^{\circ}\right.$ angle) for an additional 20 min. HRV for supine and inclined positions (that is, the tilt test) was recorded using an electrocardiogram (AD Instruments, Sydney, Australia), and a time series of RR interval (RRi) was obtained. This RRi time series was divided into continuous segments of 200 beats and overlapped with the previous series of 100 beats. The mean and variance of each segment were calculated, and these values were submitted to an auto-regressive spectral analysis as described previously. ${ }^{24-26}$ The oscillatory components in stationary segments (beat-to-beat RRi) were calculated based on the Levinson-Durbin recursion in accordance with Akaike's criteria. ${ }^{24}$ This method allows for the central frequency and influence of each relevant oscillatory component in the RRi series to be automatically quantified. The oscillatory components were classified as low frequency (LF; $0.04-0.15 \mathrm{~Hz}$ ) or high frequency (HF; $0.15-$ $0.5 \mathrm{~Hz}$ ). The power of these components on RRi variability was calculated as the variability of LF and HF in percent values, considering the total power and following the subtraction of the very LF $(<0.04 \mathrm{~Hz})$, and the power was expressed in normalized units. This normalization procedure minimizes the effect on the absolute values of LF and HF variability that result from the change in total power. ${ }^{24-26}$ Finally, the LF/HF rate was calculated to establish an index of autonomic modulation.

\section{Statistical analysis}

Data are presented as the mean \pm the s.e. of the mean. Comparisons between the mean values in the three groups were performed using a one-way analysis of variance followed by Tukey's post-hoc test. Comparisons between the two mean values of the same variable before and after treatment within the same group were performed using the Student's $t$-test for paired samples. Comparisons between the various mean values within the same group were performed using a one-way analysis of variance followed by either Bonferroni's post-hoc test to compare the variable before and during the treatment or Tukey's test for comparisons between groups. Differences were defined as statistically significant at $P<0.05$.

\section{RESULTS}

Table 1 lists all of the characteristics of the subjects, metabolic values, hemodynamic values and LVM indexes before and after the 15-week aerobic physical training. No significant differences in age, height, initial and final weight, body mass index, percent body fat and metabolic values were observed between the groups. However, physical training increased the $\mathrm{VO}_{2 \text { peak }}$ in all groups. Basal HR decreased at the end of the protocol and was similar before and after physical training in all groups. AP was different in all groups before physical training. The HT group had the highest AP values, and the NT group had the lowest systolic, diastolic and mean values. All variables were reduced after physical training compared with baseline values in the HT group, but remained higher than the other groups. At the end of the protocol, the values for diastolic and MAP were similar between the ACEi and NT groups. All groups demonstrated different LVM values before physical training; the NT group presented the lowest values, and the HT group displayed the highest values. Physical training did not alter the LVM index in the NT group, but a reduction in the LVM index was achieved in the other groups. Physical training had a significant impact in the HT group, whose values were higher than the trained NT group.

\section{Spectral analysis of HRV (supine rest position)}

The HT group displayed an increase in both total variance and LF oscillations (absolute and normalized units) and a decrease in HF oscillations (normalized units) compared with the other groups (Table 2). Moreover, this group also demonstrated a significant increase in the LF/HF ratio. Physical training did not affect HRV in the supine rest position in the NT group. However, the ACEi group presented a significant reduction in the total variance $(1842 \pm 281 \mathrm{vs}$. $\left.1314 \pm 104 \mathrm{~ms}^{2}\right)$ and HF oscillations in absolute units $(328 \pm 67$ vs. $213 \pm 41 \mathrm{~ms}^{2}$ ). In addition, physical training significantly improved HRV in the HT group, which was characterized by a reduction in LF oscillations in absolute $\left(774 \pm 104 v s .371 \pm 50 \mathrm{~ms}^{2}\right)$ and normalized ( $68 \pm 3$ vs. $56 \pm 4 \%)$ units, an increase in HF oscillations in normalized units $(32 \pm 3$ vs. $44 \pm 4 \%)$ and a reduction in the LF/HF ratio $(2.5 \pm 0.4$ vs. $1.3 \pm 0.2)$.

\section{Spectral analysis of HRV (Tilt test)}

Figure 1 presents representative spectra of RRi for all groups in the supine position and during the tilt test before and after physical training. Table 3 shows the results of the tilt test in volunteers before the 15-week physical training protocol (sedentary). The autonomic modulatory responses to the tilt test were not different between the NT and ACEi groups. In contrast, the HT group exhibited a greater reduction in total variance, a smaller increase in LF oscillations in percent values (Figure 2a), and an increase in the LF/HF ratio. After physical training (Table 3 and Figure 2), all groups demonstrated improved cardiac modulatory responses to the tilt test with no significant differences between groups. However, a smaller reduction in total variance was observed in the ACEi group. 
Table 1 Clinical characteristics of study volunteers participants before (Sedentary) and after physical training (Trained)

\begin{tabular}{|c|c|c|c|c|c|c|}
\hline & \multicolumn{2}{|c|}{ NT group } & \multicolumn{2}{|c|}{ HT group } & \multicolumn{2}{|c|}{ ACEi group } \\
\hline & Sedentary & Trained & Sedentary & Trained & Sedentary & Trained \\
\hline \multicolumn{7}{|l|}{ Subjects characteristics } \\
\hline Age, years & $44 \pm 4$ & & $44 \pm 3$ & & $45 \pm 4$ & \\
\hline Height, m & $1.62 \pm 0.02$ & & $1.64 \pm 0.03$ & & $1.68 \pm 0.03$ & \\
\hline Weight, kg & $74.7 \pm 4.4$ & $73.6 \pm 3.3$ & $80.1 \pm 4.1$ & $77.6 \pm 3.6$ & $85.2 \pm 4.9$ & $83.4 \pm 2.8$ \\
\hline $\mathrm{BMI}, \mathrm{kg} \mathrm{m}^{-2}$ & $28.4 \pm 0.6$ & $28.0 \pm 1.5$ & $29.8 \pm 1.6$ & $28.8 \pm 1.2$ & $29.4 \pm 1.1$ & $28.5 \pm 0.9$ \\
\hline Fat mass, \% & $31.3 \pm 1.2$ & $30.3 \pm 0.8$ & $31.5 \pm 1.4$ & $30.7 \pm 1.9$ & $32.0 \pm 1.8$ & $31.1 \pm 2.1$ \\
\hline \multicolumn{7}{|l|}{ Metabolic values } \\
\hline $\mathrm{VO}_{2 \text { peak }}, \mathrm{ml} \mathrm{kg}^{-1} \mathrm{~min}^{-1}$ & $29.2 \pm 1.5$ & $32.9 \pm 1.1^{*}$ & $27.1 \pm 0.9$ & $30.8 \pm 1.8^{\dagger}$ & $26.4 \pm 1.4$ & $33.1 \pm 2.3^{\#}$ \\
\hline Total Cholesterol, $\mathrm{mg} \mathrm{dl}^{-1}$ & $207.4 \pm 9.6$ & $199.8 \pm 12.2$ & $215.7 \pm 8.3$ & $202.1 \pm 10.6$ & $224.1 \pm 13.7$ & $199.1 \pm 11.7$ \\
\hline $\mathrm{HDL}, \mathrm{mg} \mathrm{dl}^{-1}$ & $52.5 \pm 4.2$ & $57.7 \pm 4.8$ & $48.4 \pm 6.2$ & $49.6 \pm 5.4$ & $47.8 \pm 3.9$ & $51.1 \pm 1.4$ \\
\hline $\mathrm{LDL}, \mathrm{mg} \mathrm{dl}^{-1}$ & $138.6 \pm 11.5$ & $130.8 \pm 8.4$ & $144.1 \pm 6.9$ & $129.7 \pm 10.7$ & $143.2 \pm 5.5$ & $125.7 \pm 5.6$ \\
\hline Triglycerides, mg dl-1 & $135.1 \pm 12.6$ & $112.3 \pm 14.7$ & $123.4 \pm 23.5$ & $109.1 \pm 17.5$ & $134.6 \pm 17.6$ & $126.1 \pm 18.7$ \\
\hline Glucose, $\mathrm{mg} \mathrm{dl}^{-1}$ & $94.4 \pm 4.2$ & $90.3 \pm 3.8$ & $93.2 \pm 6.7$ & $90.4 \pm 4.5$ & $95.3 \pm 4.6$ & $91.7 \pm 2.2$ \\
\hline \multicolumn{7}{|l|}{ Baseline cardiovascular values } \\
\hline Left ventricular mass, $\mathrm{g} \mathrm{m}^{-2}$ & $125 \pm 2.1$ & $123 \pm 2.8$ & $143 \pm 3.6^{*}$ & $133 \pm 2.0^{\S}$ & $135 \pm 2.9^{*, \dagger}$ & $129 \pm 3.8$ \\
\hline Heart Rate, bpm & $70 \pm 2$ & $64 \pm 2 *$ & $71 \pm 3$ & $63 \pm 2^{\dagger}$ & $71 \pm 3$ & $65 \pm 2^{\#}$ \\
\hline Systolic AP, mm Hg & $118 \pm 3$ & $117 \pm 3$ & $142 \pm 4^{*}$ & $134 \pm 2^{\S, \dagger}$ & $132 \pm 3^{*, \dagger}$ & $126 \pm 3 \S$ \\
\hline Diastolic AP, mm Hg & $79 \pm 2$ & $78 \pm 2$ & $94 \pm 3^{*}$ & $87 \pm 2^{\S, \dagger}$ & $86 \pm 2^{*, \dagger}$ & $80 \pm 2^{\bullet, \#}$ \\
\hline Mean AP, mm Hg & $94 \pm 3$ & $94 \pm 2$ & $113 \pm 3^{*}$ & $106 \pm 1^{\S, \dagger}$ & $104 \pm 2^{*, \dagger}$ & $98 \pm 2^{\bullet, \#}$ \\
\hline
\end{tabular}

Abbreviations: ACEi, angiotensin-converting enzyme inhibitor; AP, arterial pressure; BMI, body mass index; HDL, high-density lipoprotein; HT, hypertensive; LDL, low-density lipoprotein. All values were expressed as mean \pm s.e.m. ${ }^{*} P<0.05$ vs. normotensive group (NT) sedentary; ${ }^{\S} P<0.05$ vs. normotensive group (NT) trained; ${ }^{\dagger} P<0.05$ vs. hypertensive group (HT) sedentary; $\because P<0.05$ vs. hypertensive group (HT) trained; ${ }^{\#} P<0.05$ vs. angiotensin-converting enzyme inhibitor group (ACEi) sedentary.

Table 2 Spectral parameters of R-R interval (RRi) calculated from time series obtained all sedentary volunteers in supine position and during the tilt test

\begin{tabular}{|c|c|c|c|c|c|c|}
\hline & \multicolumn{2}{|c|}{ NT Group } & \multicolumn{2}{|c|}{ HT Group } & \multicolumn{2}{|c|}{ ACEi Group } \\
\hline & Supine & Tilt & Supine & Tilt & Supine & Tilt \\
\hline RRi, ms & $846 \pm 31$ & $761 \pm 27^{*}$ & $874 \pm 29$ & $755 \pm 23^{\dagger}$ & $851 \pm 31$ & $788 \pm 18^{\#}$ \\
\hline \multicolumn{7}{|c|}{ Spectral Parameters; RRi } \\
\hline Variance, $\mathrm{ms}^{2}$ & $1953 \pm 281$ & $1618 \pm 284$ & $2768 \pm 209 *$ & $1366 \pm 154^{\dagger}$ & $1842 \pm 281^{\dagger}$ & $1207 \pm 108^{\#}$ \\
\hline $\mathrm{LF}, \mathrm{ms}^{2}$ & $369 \pm 92$ & $282 \pm 61$ & $774 \pm 104 *$ & $518 \pm 88^{\dagger}$ & $288 \pm 64^{\dagger}$ & $263 \pm 51$ \\
\hline LF, nu & $49 \pm 6$ & $70 \pm 5^{*}$ & $68 \pm 3^{*}$ & $85 \pm 3^{\dagger}$ & $50 \pm 4^{\dagger}$ & $70 \pm 3^{\#}$ \\
\hline $\mathrm{HF}, \mathrm{ms}^{2}$ & $344 \pm 69$ & $101 \pm 19^{*}$ & $337 \pm 44$ & $74 \pm 13^{\dagger}$ & $328 \pm 67$ & $114 \pm 24^{\#}$ \\
\hline HF, nu & $51 \pm 6$ & $30 \pm 5^{*}$ & $32 \pm 3^{*}$ & $15 \pm 3^{\dagger}$ & $50 \pm 4^{\dagger}$ & $30 \pm 3^{\#}$ \\
\hline LF/HF ratio & $1.3 \pm 0.3$ & $3.7 \pm 0.9^{*}$ & $2.5 \pm 0.4^{*}$ & $9.1 \pm 2.8^{\dagger}$ & $1.2 \pm 0.2^{\dagger}$ & $2.7 \pm 0.4^{\#}$ \\
\hline
\end{tabular}

Abbreviations: ACEi, angiotensin-converting enzyme inhibitor; HF, high frequency; HT, hypertensive; LF, low frequency; NT, normotensive.

All values were expressed as mean \pm s.e.m. ${ }^{*} P<0.05$ vs. normotensive group (NT) supine; ${ }^{\dagger} P<0.05$ vs. hypertensive group (HT) supine; ${ }^{\#} P<0.05$ vs. angiotensin-converting enzyme inhibitor group (ACEi) supine. 'nu' indicates normalized units.

\section{DISCUSSION}

This study demonstrated that aerobic physical training may enhance autonomic modulatory responses to the tilt test independently of the presence of hypertension or pharmacological treatment with an ACEi. Additionally, our study also demonstrated that aerobic physical training reduced arterial pressure in untreated HT volunteers and further reduced AP values in association with Enalapril compared with the use of either treatment alone.

Arterial hypertension is often accompanied by an impairment of cardiovascular autonomic control, which is characterized by an increase in sympathetic autonomic activity and a decrease in vagal autonomic activity assessed by different approaches of peripheral sympathetic nervous activity, such as baroreflex sensitivity and HRV analysis. ${ }^{21,27-29}$ Many studies have demonstrated that HT volunteers exhibit a reduction in HRV that is associated with a decrease in vagal autonomic modulation and an increase in sympathetic modulation. ${ }^{30-32}$

Our study demonstrated that untreated HT volunteers presented an increase in HVR that was characterized by an increase in total variance in the RRi compared with NT and HT volunteers treated with Enalapril. Although this increase appears paradoxical, it was the result of an increase in sympathetic autonomic modulation because the LF oscillations in these volunteers were notably high. A reduction in HF oscillations was also observed in this group, which further contributed to the increase in LF/HF rate and indicated a greater predominance of sympathetic activity in the autonomic balance. 

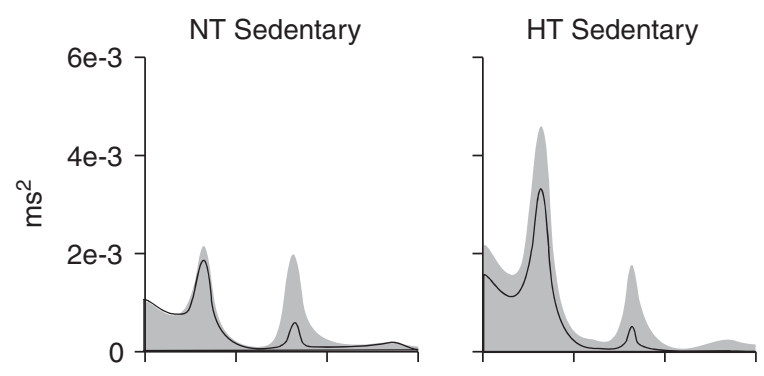

ACEi Sedentary
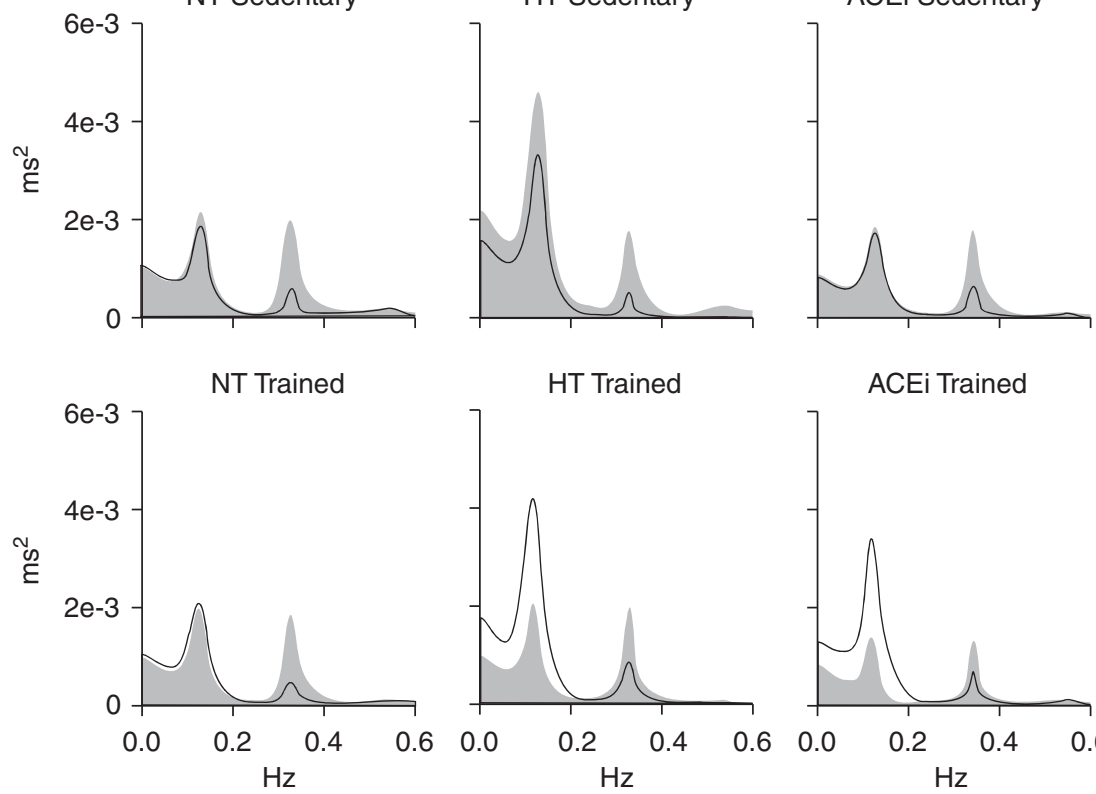

ACEi Trained

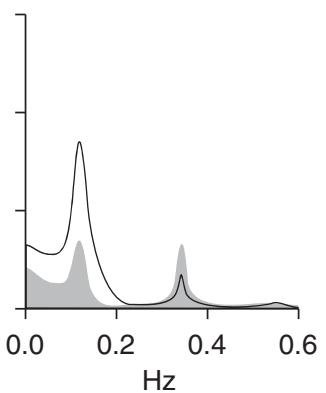

Figure 1 Examples of representative autoregressive spectra calculate from series of $R-R$ interval $\left(\mathrm{ms}^{2}\right)$ during supine position (black line) and tilt test (gray fill) in sedentary (top) and trained (bottom) volunteers. Normotensive (NT); Hypertensive (HT); and angiontensin-converting enzyme inhibitor (ACEi) treated volunteer.

Table 3 Spectral parameters of $\mathbf{R}-\mathbf{R}$ interval (RRi) calculated from time series obtained all trained volunteers in supine position and during the tilt test

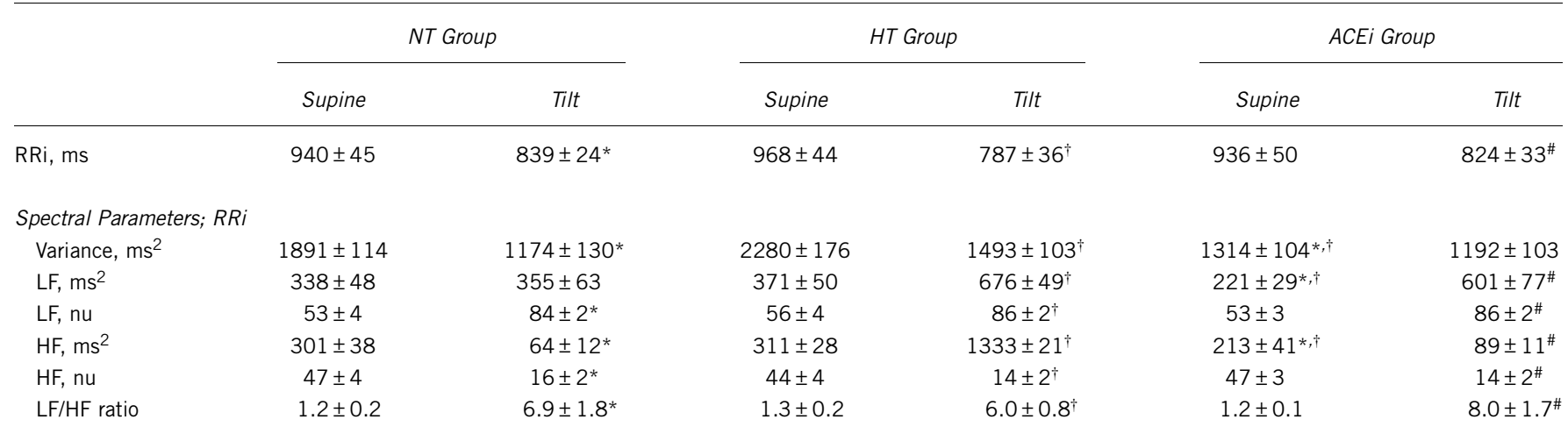

Abbreviations: ACEi, angiotensin-converting enzyme inhibitor; HF, high frequency; HT, hypertensive; LF, low frequency; NT, normotensive.

All values were expressed as mean \pm s.e.m. ${ }^{*} P<0.05$ vs. normotensive group (NT) supine; ${ }^{*} P<0.05$ vs. hypertensive group (HT) supine; ${ }^{\#} P<0.05$ vs. angiotensin-converting enzyme inhibitor group (ACEi) supine. 'nu' indicates normalized units.

HRV can be evaluated in different situations, and the tilt test assesses autonomic balance rearrangement during postural stimuli. ${ }^{33,34}$ In our study, the tilt test revealed that untreated HT volunteers had greater increases in the absolute values of LF oscillations but low responses in percent values. However, the percent values of the HF band were similar to other groups despite the lower absolute values. This finding suggests that the impaired cardiac autonomic control is mainly the result of the sympathetic autonomic component. $^{31-35}$

Subjects in the ACEi group exhibited a significant reduction in arterial pressure compared with the HT groups and no difference in cardiac autonomic modulation compared with NT volunteers during the tilt test. This observation suggests that treatment with Enalapril re-established the cardiac autonomic modulation that was impaired by hypertension. Indeed, ACEis and AT1 receptor antagonists promote an improvement in autonomic function in different contexts, including baroreflex sensitivity evaluation and HRV analysis. ${ }^{11,28,30,36,37}$ The mechanisms that underlie ACEi improvement of HRV modulation have not been elucidated, but ACEis and/or AT1receptor antagonists primarily affect neural sites that are involved in the cardiovascular regulation, such as the solitary tract nucleus, rostral ventrolateral medulla, paraventricular nucleus and area postrema. However, the identification of the exact mechanism of action has been hindered by the complex interaction of the sites involved in cardiovascular control. ${ }^{30,38-40}$

Similar to Enalapril, physical training also reduces arterial pressure. The combination of Enalapril and physical training enhanced the reduction of $\mathrm{AP}$, which reached values that were similar to the NT volunteers. The effect of physical exercise in HT patients with mild-tomoderate arterial hypertension has been described previously. ${ }^{41}$ Our results corroborate earlier studies and suggest that pharmacological therapy with physical exercise promotes better outcomes. 

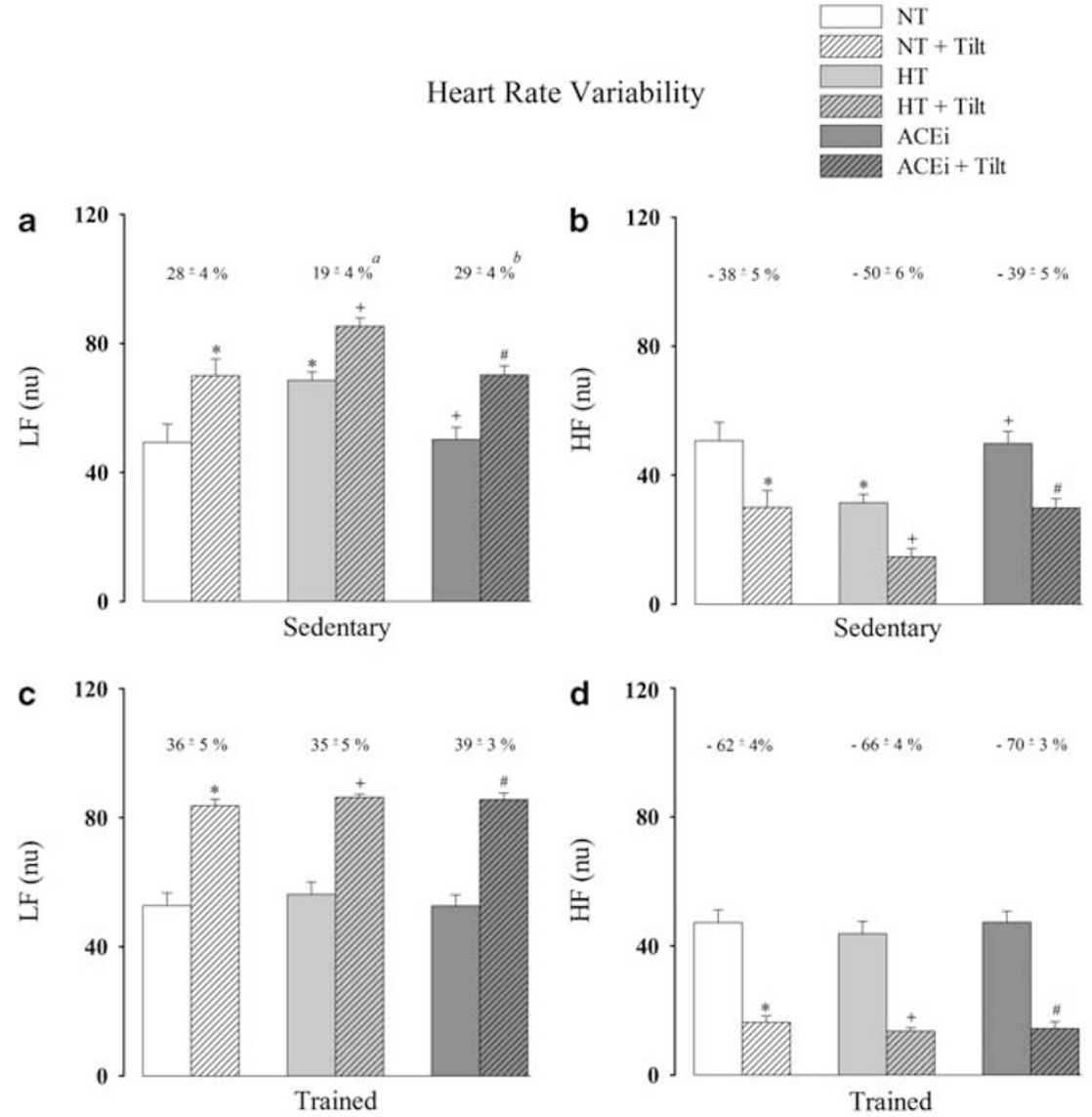

Figure 2 Spectral power density of R-R interval in low (LF) and high frequency (HF) bands in normalizes units (nu) during supine position and after the tilt test (with their respective \% change) calculated from time series in all volunteers groups before (a, b) and after (c, d) the aerobic physical training. Normotensive (NT group), Hypertensive (HT group) and Angiotensin-converting enzyme inhibitor treated (ACEi group) volunteers. ${ }^{*} P<0.05$ vs. NT group; ${ }^{+} P<0.05$ vs. HT group; ${ }^{\#} P<0.05$ vs. ACEi group; ${ }^{a} P<0.05$ vs. NT group (\% change after tilt test); and ${ }^{b} P<0.05$ vs. HT group ( $\%$ change after tilt test).

Physical training also promoted significant changes in HRV despite a more intensive and independent activity on angiotensin II blockade. Our results demonstrated that aerobic physical training improved the autonomic modulatory responses of HRV in all groups in addition to the reestablishment of the modulatory parameters in untreated hypertension volunteers, whose values were similar to those of NT volunteers. Therefore, the improvement in spectral parameters during the tilt test was similar between groups. Interestingly, aerobic physical training did not alter basal cardiac autonomic modulation in NT or HT volunteers treated with Enalapril. This result emphasizes the importance of the evaluation of cardiac autonomic modulation in different situations (for example, using the tilt test) to provide important elements of the capacity for autonomic balance rearrangement during therapeutic interventions.

Our findings support the importance of physical training for therapeutic and preventive purposes in the treatment of hypertension itself and account for important alterations in cardiac autonomic adjustments. ${ }^{18,19}$ Physical exercise may promote adjustments in cardiovascular control sites, such as the hypothalamus, solitary tract nucleus and the rostral ventrolateral medulla. These adaptations may occur via neural remodeling or endogenous factors, such as nitric oxide. ${ }^{20,42-46}$ These adaptations would promote a decrease in sympathetic autonomic influence and an increase in the participation of the vagal autonomic component in cardiac control to improve the autonomic balance. Additionally, physical exercise may also exacerbate the influence of angiotensin II on the modulation activity in specific neural sites, ${ }^{45}$ but further investigation is required.

Some limitations of our study should be addressed. Firstly, AP and HR were not consecutively assessed in all groups before treatment with Enalapril in the ACEi group. Secondly, a follow-up study of HRV during this same interval would have provided information on autonomic modulation in the ACEi group before treatment and allowed for a comparison of values from the HR group. Further longitudinal studies should be performed using the parameters of the present study to investigate the effects of other anti-HT drugs and physical training regimens.

In conclusion, our findings demonstrate that physical exercise is important for therapeutic and preventive purposes and serves as a co-adjuvant in the treatment of hypertension. This approach promotes cardiac autonomic modulation in HT volunteers independently of treatment with ACEis.

\section{CONFLICT OF INTEREST}

The authors declare no conflict of interest.

\section{ACKNOWLEDGEMENTS}

This study was supported by FAPESP (2008/07561-0). 
1 Kannel WB. Framingham study insights into hypertensive risk of cardiovascular disease. Hypertens Res 1995; 18: 181-196.

2 Kearneya PM, Wheltona M, Reynoldsa K, Wheltona PK, He J. Worldwide prevalence of hypertension: a systematic review. J Hypertens 2004; 22: 11-19.

3 Whelton PK. Epidemiology of hypertension. Lancet 1994; 344: 101-106.

4 He J, Bazzano LA. Effects of lifestyle modification on treatment and prevention of hypertension. Curr Opin Neph Hyp 2000; 9: 267- 271.

5 Cupples LA, D'agostino RB. Section 34: some risk factors related to the annual incidence of cardiovascular disease and death in pooled repeated biennial measurements. In: Kannel WB, Wolf PA, Garrison RJ, (eds) Framingham Heart Study: 30 Year Follow FollUp. Bethesda 1987, pp 1- 22.

6 Perret-Guillaume $C$, Joly L, Jankowski P, Benetos A. Benefits of the RAS blockade: clinical evidence before the ONTARGET study. J Hypertens 2009; 27 (suppl 2): S3-S7.

7 Duprez DA. Role of the renin-angiotensin-aldosterone system in vascular remodeling and inflammation: a clinical review. J Hypertens 2006; 24: 983-991.

8 Mizuno Y, Jacob RF, Mason RP. Effects of calcium channel and renin-angiotensin system blockade on intravascular and neuro-hormonal mechanisms of hypertensive vascular disease. Am J Hypertens 2008; 21: 1076-1085.

9 Zhang GQ, Zhu Z, Zhang W. Inhibitory effect of antihypertensive drugs on calcineurin in cardiomyocytes. Am J Hypertens 2009; 22: 132-136.

10 Ball SG. The sympathetic nervous system and converting enzyme inhibition. $J$ Cardiovasc Pharmacol 1989; 13: S17-S21.

11 Karas M, Lacourcière Y, Leblanc AR, Nadeau R, Dubé B, Florescu M, Lamarre-Cliche M, Poirier L, Larochelle P, De Champlain J. Effect of the renin-angiotensin system or calcium channel blockade on the circadian variation of heart rate variability, blood pressure and circulating catecholamines in hypertensive patients. J Hypertens 2005; 23: 1137-1139.

12 Dias da Silva VJ, Montano N, Salgado HC, Fazan Júnior R. Effects of long-term angiotensin converting enzyme inhibition on cardiovascular variability in aging rats. Auton Neurosci 2006; 124: 49-55.

13 Fagard RH, Cornelissen VA. Effect of exercise on blood pressure control in hypertensive patients. Eur J Card Prev Rehab 2007; 14: 12-17.

14 Whelton SP, Chin A, Zin Z, He J. Effects of aerobic exercise on blood pressure: a metaanalysis of randomized controlled trials. Ann Intern Med 2002; 136: 493-503.

15 Izdebska E, Cybulska I, Izdebski J, Makowiecka-Ciesla M, Trebski A. Effects of Moderate Physical Training on Blood Pressure Variability and Hemodynamic Pattern in Mildly Hypertensive Subjects. J Physiol Pharmcol 2004; 55: 713-724.

16 Cornelissen VA, Fagard RH. Effects of endurance training on blood pressure, blood pressure-regulating mechanisms, and cardiovascular risk factors. Hypertension 2005; 46: 667-675.

17 Kokkinos P, Nrayan P, Papademetriou V. Exercise as hypertension therapy. Cardiol Clin 2001; 19: 507-516

18 Laterza MC, de Matos L, Trombetta Cl, Braga AMW, Roveda F, Alves MJ, Krieger EM, Negrão CE, Rondon MU. Exercise Training Restores Baroreflex Sensitivity in NeverTreated Hypertensive Patients. Hypertension 2007; 49: 1298-1306.

19 Carter JB, Banister EW, Blaber AP. Effect of endurance exercise on autonomic control of heart rate. Sports Med 2003; 33: 33-46.

20 Souza HCD, de Araujo JE, Martins-Pinge MC, Cozza IC, Martins-Dias DP. Nitric oxide synthesis blockade reduced the baroreflex sensitivity in trained rats. Auton NeurosCi Basic Clin 2009; 150: 38-44.

21 Collier SR, Kanaley JA, Carhart Jr R, Frechette V, Tobin MM, Bennett N, Luckenbaugh AN, Fernhall B. Cardiac autonomic function and baroreflex changes following 4 weeks of resistance versus aerobic training in volunteers with pre-hypertension. Acta Physiol (Oxf) 2009; 195: 339-348.

22 Sloan RP, Shapiro PA, DeMeersman RE, Bagiella E, Brondolo EN, McKinley PS, Slavov I, Fang Y, Myers MM. The effect of aerobic training and cardiac autonomic regulation in young adults. Am J Public Health 2009; 99: 921-928.

23 Piotrowicz E, Baranowski R, Piotrowska M, Zieliñski T, Piotrowicz R. Variable effects of physical training of heart rate variability, heart rate recovery, and heart rate turbulence in chronic heart failure. Pacing Clin Electrophysiol 2009; 32(suppl 1): S113-S115.

24 Malliani A, Pagani M, Lombardi F, Cerutti S. Cardiovascular neural regulation explored in the frequency domain. Circulation 1991; 84: 482-492.
25 Rubini R, Porta A, Baselli G, Cerutti S, Paro M. Power spectrum analysis of cardiovascular variability monitored by telemetry in conscious unrestrained rats. J Auton Nerv Syst 1993; 45: 181-190.

26 Task Force of the European Society of Cardiology and the North American Society of Pacing and Electrophysiology. Heart rate variability: standards of measurement, physiological interpretation and clinical use. Circulation 1996; 93: 1043-1065.

27 Schlaich MP, Lambert E, Kaye DM, Krozowski Z, Campbell DJ, Lambert G, Hastings J, Aggarwal A, Esler MD. Sympathetic augmentation in hypertension: role of nerve firing, norepinephrine reuptake, and Angiotensin neuromodulation. Hypertension 2004; 43: $169-175$.

28 Neumann J, Ligtenberg G, Klein IH, Boer P, Oey PL, Koomans HA, Blankestijn PJ. Sympathetic hyperactivity in hypertensive chronic kidney disease patients is reduced during standard treatment. Hypertension 2007; 49: 506-510.

29 Grassi G. Assessment of sympathetic cardiovascular drive in human hypertension: achievements and perspectives. Hypertension 2009; 54: 690-697.

30 Townend JN, al-Ani M, West JN, Littler WA, Coote JH. Modulation of cardiac autonomic control in humans by angiotensin II. Hypertension 1995; 25: 1270-1275.

31 Pagani M, Lucini D. Autonomic dysregulation in essential hypertension: insight from heart rate and arterial pressure variability. Auton Neurosci 2001; 90: 76-82.

32 Lucini D, Mela GS, Malliani A, Pagani M. Impairment in cardiac autonomic regulation preceding arterial hypertension in humans: insights from spectral analysis of beat-bybeat cardiovascular variability. Circulation 2002; 106: e9051.

33 Frohlich ED, Tarazi RC, Ulrych M, Dustan HP, Page IH. Tilt test for investigating a neural component in hypertension: Its correlation with clinical characteristics. Circulation 1967; 36: 387-393.

34 Goldstein RE, Beiser GD, Stampfer M, Epstein SE. Impairment of autonomically mediated heart rate control in patients with cardiac dysfunction. Circ Res 1975; 36: 571-578.

35 Pavithran P, Mithun R, Jomal M, Nandeesha H. Heart rate variability in middleaged men with new-onset hypertension. Ann Noninvasive Electrocardiol 2008; 13 : 242-248.

36 Petretta M, Bonaduce D, Marciano F, Bianchi V, Valva G, Apicella C, De Luca N, Gisonni P. Effect of 1 year of lisinopril treatment on cardiac autonomic control in hypertensive patients with left ventricular hypertrophy. Hypertension 1996; 27(3 Pt 1): 330-338.

37 Chern CM, Hsu HY, Hu HH, Chen YY, Hsu LC, Chao AC. Effects of atenolol and losartan on baroreflex sensitivity and heart rate variability in uncomplicated essential hypertension. Cardiovasc Pharmacol 2006; 47: 169-174.

38 Matsukawa S, Reid IA. Role of the area postrema in the modulation of the baroreflex control of heart rate by angiotensin II. Circ Res 1990; 67: 1462-1473.

39 DiBona GF. Peripheral and central interactions between the renin-angiotensin system and the renal sympathetic nerves in control of renal function. Ann N Y Acad Sc 2001; 940: 395-406.

40 Tan PS, Killinger S, Horiuchi J, Dampney RA. Baroreceptor reflex modulation by circulating angiotensin II is mediated by AT 1 receptors in the nucleus tractus solitarius. Am J Physiol Regul Integr Comp Physiol 2007; 293: R2267-R2278.

41 Manfredini F, Malagoni AM, Mandini S, Boari B, Felisatti M, Zamboni P, Manfredini R. Sport therapy for hypertension: why, how, and how much? Angiology 2009; 60: 207-216.

42 Zucker IH, Schultz HD, Li YF, Wang Y, Wang W, Patel KP. The origin of sympathetic outflow in heart failure: the roles of angiotensin II and nitric oxide. Prog Biophys $\mathrm{Mol}$ Biol 2004; 84: 217-232.

43 Zheng H, Li YF, Cornish KG, Zucker IH, Patel KP. Exercise training improves endogenous nitric oxide mechanisms within the paraventricular nucleus in rats with heart failure. Am J Physiol Heart Circ Physiol 2005; 288: H2332-H2341.

44 Mueller PJ, Foley CM, Hasser EM. Hindlimb unloading alters nitric oxide and autonomic control of resting arterial pressure in conscious rats. Am J Physiol Regul Integr Comp Physiol 2005; 289: R140-R147.

45 Felix JV, Michelini LC. Training-induced pressure fall in spontaneously hypertensive rats is associated with reduced angiotensinogen mRNA expression within the nucleus tractus solitarii. Hypertension 2007; 50: 780-785.

46 De Abreu SB, Lenhard A, Mehanna A, de Souza HC, Correa FM, Hasser EM, MartinsPinge MC. Role of paraventricular nucleus in exercise training-induced autonomic modulation in conscious rats. Auton Neurosci 2009; 148: 28-35. 